\title{
Una tendencia de la arquitectura moderna en América Latina, basada en la Geometría revolucionaria de la iglesia de San Francisco de Asís
}

\section{A trend of modern architecture in Latin America, based on the revolutionary geometry of the church of San Francisco de Asís}

\author{
Yannette Díaz-Umaña ${ }^{a}$, Julio Alfredo Delgado-Rojas ${ }^{\mathrm{b}}$, Mawency Vergel-Ortega ${ }^{\mathrm{c}}$ \\ aMagister en Gestión Urbana, yannettedu@ufps.edu.co, https://orcid.org/0000-0003-4582-1593, Universidad Francisco de Paula Santander, Cúcuta, \\ Colombia \\ bMagister en Educación Matemática, julioalfredo@ufps.edu.co, https://orcid.org/0000-0001-6944-832X, Universidad Francisco de Paula Santander, \\ Cúcuta, Colombia \\ 'Doctora en Educación, mawency@ufps.edu.co, https://orcid.org/0000-0001-8285-2968, Universidad Francisco de Paula Santander, Cúcuta, Colombia
}

Forma de citar: Díaz-Umaña, Y. ., Delgado-Rojas, J. A. ., \& Vergel-Ortega, M., (2021). Una tendencia de la arquitectura moderna en América Latina, basada en la Geometría revolucionaria de la iglesia de San Francisco de Asís. Eco Matemático, 12 (2), 6-13

Recibido: 3 deFebrero de 2021

Aceptado:2 de abril de 2021

\section{Palabras clave}

Arquitectura moderna

sagrada,

geometría,

curvas cónicas,

arco catenario

\section{Keywords}

Modern sacred architecture, geometry, conical curves, catenary arch
Resumen: Este artículo se deriva de la investigación en arquitectura sagrada, bajo la dirección de los programas de matemáticas y arquitectura de la Universidad Francisco de Paula Santander, cuyo objetivo, en este caso fue analizar la arquitectura y la geometría de la iglesia de San Francisco de Asís en Brasil por cuanto su innovación tipológica haciendo uso de la geometría. En este proyecto con el estudio descriptivo, se analiza las variables del diseño arquitectónico para la arquitectura moderna sagrada, con especial énfasis en las condiciones particulares que hacen de la cubierta una estructura, liviana, resistente y propicia para generar una espacialidad libre de apoyos conforme a los requerimientos espaciales y estéticos de mediados del siglo XX. En este análisis se pudo observar, que, con el uso del arco catenario, se le otorga las cualidades estructurales, estéticas y espaciales que permiten valorar un revolucionario diseño.

Abstract: This article is derived from research in sacred architecture, under the direction of the mathematics and architecture programs of the UFPS, whose objective, in this case, was to analyze the architecture and geometry of the church of San Francisco de Asís in Brazil as its typological innovation making use of geometry. In this project with the descriptive study, the variables of the architectural design for sacred modern architecture are analyzed, with special emphasis on the particular conditions that make the roof a structure, light, resistant and conducive to generating a spatiality free of supports according to the spatial and aesthetic requirements of the mid-twentieth century. In this analysis, it was observed that, with the use of the catenary arch, it is given the structural, aesthetic and spatial qualities that allow a revolutionary design to be valued.

*Autor para correspondencia yannettedu@ufps.edu.co

https://doi.org/10.22463/17948231.3074 


\section{Introducción}

Este artículo se deriva de la investigación en arquitectura sagrada con propósitos de integración curricular desarrollada entre el programa de matemáticas y el programa de arquitectura de la Universidad Francisco de Paula Santander. El caso de estudio se delimita a la obra ubicada en Belo Horizonte, la iglesia de San Francisco de Asís, diseñada por el arquitecto Oscar Niemeyer e inaugurada el año 1943, cuyo impacto la hizo un importante hito en la arquitectura moderna de Latinoamérica. Este ejemplo que brinda la oportunidad de analizar la conceptualización de la geometría vinculada al diseño de la arquitectura sagrada, de tal forma que el diseño metodológico se plateó teniendo en cuenta el enfoque de la investigación de tipo descriptivo, el interés es cualificar, y describir con un uso natural del lenguaje el objeto de estudio según Sabino (2008), dando una visión general y aproximada de su sentido de habitabilidad para la misión litúrgica.

Es importante señalar, el interés en este tipo de estudios, debido a que, la iglesia de San Francisco de Asís, es una de las primeras en Latinoamérica con membrana estructural de hormigón en perfil curvo, modelo representativo, que han sido motivo de estudio en otros contextos, constituyendo una importante contribución a la memoria mundial. Para Duque (2012) gracias a las libertades plásticas que permite el hormigón, se pudo dejar la monotonía, no obstante, aunque la iglesia es considerada la obra más importante del conjunto Pampulha, recibió numerables críticas incluso, de las autoridades eclesiásticas, por su novedosa forma. En este sentido Philippou (2013) comenta:

"Sin embargo, al tomar prestadas las formas de la arquitectura industrial para la cúpula celeste del programa eclesiástico, Niemeyer no pretendía desacralizar el espacio eclesiástico, sino apropiarse de las estructuras de hormigón armado de los programas utilitarios, e introducirlas en el reino privilegiado del arte culto. No obstante, las autoridades conservadoras de la iglesia de Minas Gerais consideraron la iglesia de San Francisco sacrílega y provocadora. Su reacción violenta se prolongó a lo largo de los años. Hasta 1959, la iglesia se negó a consagrar el edificio. Por otro lado, la iglesia de Pampulha fue el primer edificio moderno de Brasil en ser declarado monumento nacional en 1943, solo un año después de su conclusión" (pág. 11)

Se insinúa que el nacimiento de la arquitectura moderna para la arquitectura sagrada, se da en completa sincronización con el Movimiento Litúrgico y todos los fenómenos propios del siglo XIX, de orden social, económico, cultural y tecnológico, estrechamente ligados a la revolución industrial (Díaz, Delgado, \& Vergel 2021). Pero, en américa latina estos cambios tardaron un poco en hacerse habituales en los perfiles urbanos de las importantes ciudades. Particularmente en Europa, se empieza a percibir los cambios desde 1830 en Alemania, con nuevas tipologías desarrolladas en estructuras de hormigón, conceptualizadas con ideas del Movimiento Litúrgico. De esta forma se incorporaron los avances de la modernidad, reafirmado la fe en afinidad con el desarrollo científico-técnico (Díaz y Podetti 2020).

A finales del siglo XIX, las innovaciones tecnológicas, el crecimiento de la población, el comercio acelerado, incidieron directamente en la construcción, generando una realización ingenieril notable. Así mismo la producción en serie, nuevas tecnologías y la producción tradicional, desde su fuente como manufactura, se acompañaron en este periodo de eclecticismo histórico. De ahí que "...marca el paso más claro entre el pasado y el presente de la historia de la arquitectura sin el cual es impensable el nacimiento del Movimiento Moderno" (Benévolo,1980, pag.322).

En particular, se destaca en la consolidación de la arquitectura moderna, el trabajo de Adolf Loos. Su escrito Ornamento y Delito publicado en 1908 
y traducido a varios idiomas conforman una base teórica (Benévolo,1980) nutrida para las siguientes generaciones, en el sentido de buscar las formas sencillas, puras y el rechazo a la ornamentación. Le Corbusier, por su parte, con su visión purista y racionalista, geometrizó las formas, y buscó una arquitectura sencilla y más exequible en términos de dinero.

En el caso de Auguste Perret con la iglesia de Notre Dame du Raincy en 1927 y Le Corbusier con la iglesia de Notre Dame du Haut en Francia en 1950, cuyas formas plásticas en hormigón a la vista, aportaron la expresividad necesaria, para conmover al feligrés, sin el uso del ornamento. Es indudable que esta conquista del hormigón armado, es una victoria no solo francesa sino también alcanzada en otros países europeos y paulatinamente en américa. En ese sentido, De Fusco (1992) afirma:

"Pero, en realidad, nos encontramos ante un doble compromiso: si la configuración arquitectónica advierte, indudablemente, el uso del hormigón, este a su vez, siendo potencialmente capaz de producir las formas más libres y plásticas, se utiliza por motivos económicos, de más fácil calculo estático y de organización tecnológica más elemental- Renato De Fusco. 1992..." (Pág. 173 y 174)

\section{Discusión}

En efecto, el diseño de la iglesia de San Francisco de Asís, guarda estas caracterizaciones de sencillez, economía y materialidad constructiva en hormigón. Resulta interesante desde el punto de vista estético, ya que rompe con el estricto esquema tradicional de cruz latina, propio de la iglesia medieval (Delgado, Díaz, \& Vergel, 2.018). $\mathrm{Su}$ esquema responde a la actualización espacial sugerida por las mociones del Movimiento Litúrgico y a los principios de la arquitectura moderna. Lo que lleva a generalizar, de forma posterior a su construcción, que hacia la década de los 50 las principales ciudades de América Latina ya habían recibido la influencia europea de esta tendencia, pero es inevitable afirmar que el modelo de Pampulha supera las rígidas propuestas europeas basadas en el racionalismo, y que fue ésta un modelo a replicar.

Se explica de este modo, los efectos de simplificación simbólica de las iglesias en el Siglo XX. Se infiere que, la concepción formal de este templo, dispuesto tan solo por dos elementos protagónicos: la membrana con perfil curvo y la composición de azulejos de la fachada trasera, aportan junto a la piel de hormigón, cualidades de religiosidad artísticas al edificio. Esta composición retorna a los orígenes de la "construcción cristocéntrica de iglesias, al considerar el altar como punto focal de una planta central y una manufactura sencilla con materiales industriales, ajustado a los recursos del lugar, pero que en ultimas lo que busca es el efecto fenomenológico en el visitante (Díaz, Vergel, y Delgado 2020). (imagen 1)

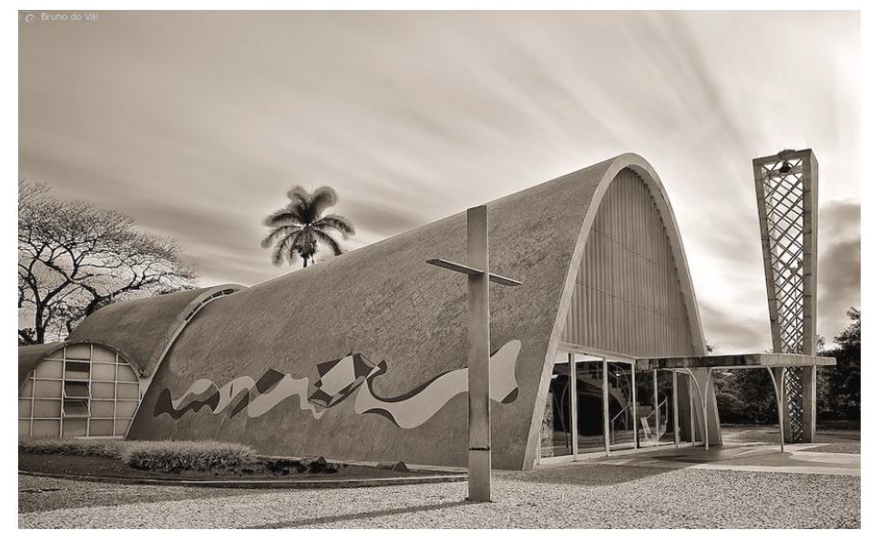

Imagen 1. Semi lateral de la iglesia de iglesia de San Francisco de Asís, Brasil

Fuente: Duque (2012)

Con esto, parece confirmar el reto que supuso el diseño arquitectónico del templo, para aportar un lenguaje que de cierta manera sea congruente con el misticismo de los antiguos templos, tal vez habitar, morar y contemplar (Heidegger, 1993), como lo consolidaba las cúpulas en los espacios sagrados. Así que la iglesia, no será solo, un objeto simplemente espacial, sino un mediador de la práctica contemplativa, en donde el recurso simbólico y artístico de esta experiencia, facilita 
descubrir el mensaje y posibilita el encuentro con Dios (Navarro, Castañeda, Peña, y Acero, 2018, pág. 205-210).

En consecuencia, el esquema planímetro, es coherente con las reformas preconciliares que emergen a finales del siglo XVIII, por la necesidad de la modernización de la Iglesia Católica y con el objetivo de renovar la vida litúrgica y la participación más dinámica de la Iglesia (León 2019). Por tanto, la conformación de un solo pabellón, en planta libre , concebido $\sin$ apoyos internos, permite una total visibilidad al altar, como una franca manera de extenderlo en el recinto, allegando el poder de la celebración a cada fiel. Otro rasgo, es la disposición del coro en un lugar estratégico, para idear la práctica y participación de la Iglesia en la alabanza, en este caso, se encuentra en los pies de la nave, en un lugar elevado, reubicando su concepción espacial original , en el presbítero, pues eran únicamente los del clero, que cantaban los oficios divinos. (imagen 2)

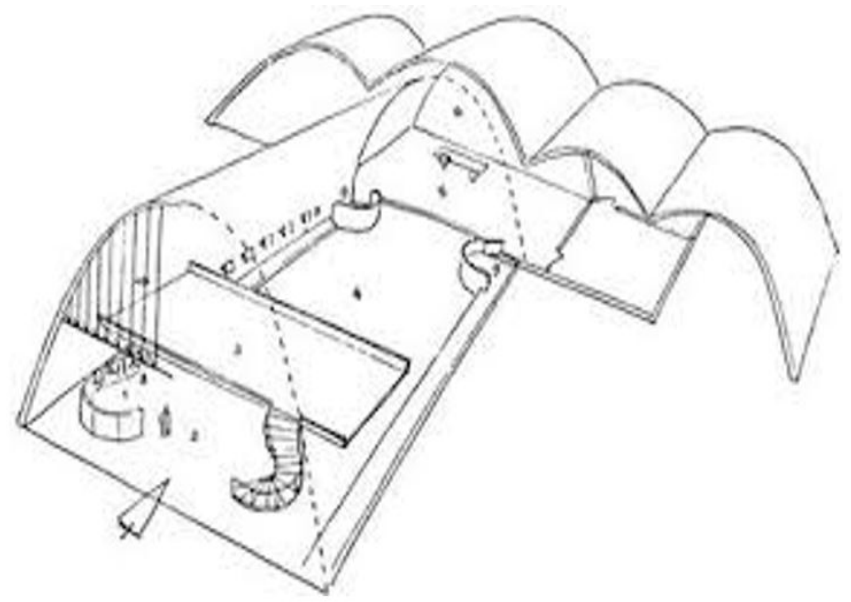

Imagen 2. Axonometría de la iglesia de San Francisco de Asís, Brasil. Descubre la relación de las cubiertas con perfil curvo con la planta libre, revelando su unidad interna

Fuente: Duque (2012)

Este envolvente en hormigón, tienen un espesor de $10 \mathrm{~cm}$, es auto portante, resistente al paso del tiempo y con especiales propiedades acústicas. El ingeniero civil Joaquim Cardozo, uno de los principales colaboradores de Niemeyer, destacó la «desaparición del muro», la irregularidad de todas las formas de la iglesia, y la manera en que cada línea de la composición está inclinada (Philippou, 2013). En general lo que se observa en esta estructura es que la tracción que padece cada punto del arco se reparte entre una componente vertical y una componente de presión que se transmite a través del propio arco hacia los cimientos, sin que se creen esfuerzos horizontales, salvo en el extremo llegando ya a los cimientos. Es esta propiedad la que hace que los arcos catenarios no necesiten apoyos laterales para sustentarse. (imagen 3)

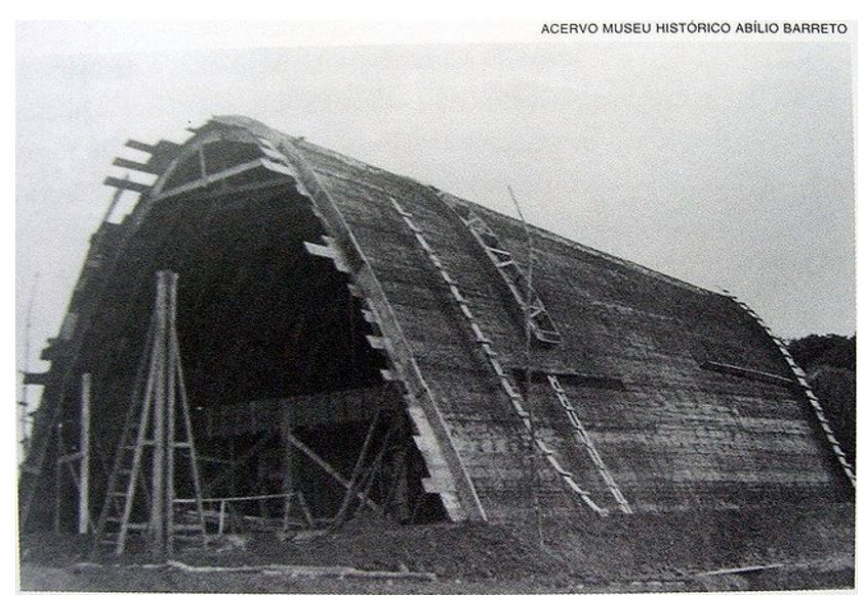

Imagen 3. Fotografía proceso constructivo. Iglesia de San Francisco de Asís, Brasil Fuente: Duque (2012)

Este distintivo revela que, aunque la obra haya pretendido romper con los esquemas estilísticos de la arquitectura sagrada medieval, se adapta el uso del azulejo, de forma análoga como el arte vitral (Vallespín, Hernández, y Cervero (2019), más que elemento decorativo, es un elemento cargado de simbolismo y religiosidad (Díaz, Vergel, y Delgado 2020), tal como era su objetivo en los templos góticos. En realidad, esta práctica con los azulejos, no es extraña en Brasil, en ocasiones los arquitectos tornaban al uso de alusiones históricas en sus diseños, como táctica respetable para engalanar la evidente novedad de sus conceptos espaciales (Téllez 1977).

De acuerdo a lo anterior, la solución estructural en aquella época de los años 40 la hace un ejemplo arquitectónico único. Como se sabe, la estructura ha sido un componente vital de la arquitectura, en este 
caso una delgada lamina curva de hormigón permite consolidar un espacio interno óptimo para la celebración litúrgica. Su estudio se concentra en su geometría, cuyas curvas logradas por el entramado de armaduras de acero. Así su geometría se relaciona, con el cono de revolución, la línea recta llamada generatriz, cuyo movimiento es fijo en un punto alrededor de un eje circular llamado directriz, que, en los cortes, se obtienen diferentes tipos de curva, de origen cónico, como la elipse, la hipérbola y la parábola (imagen 4).

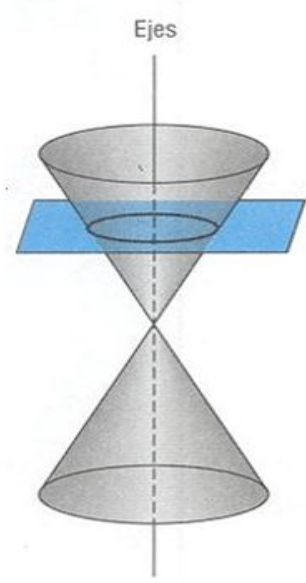

(a) Círculo

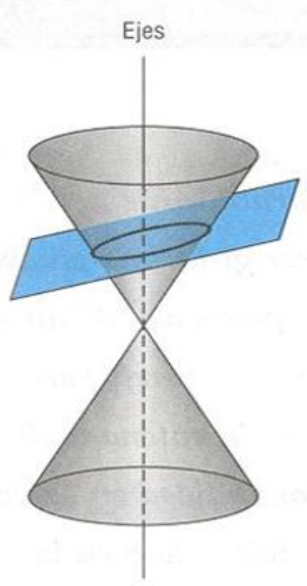

(b) Elipse

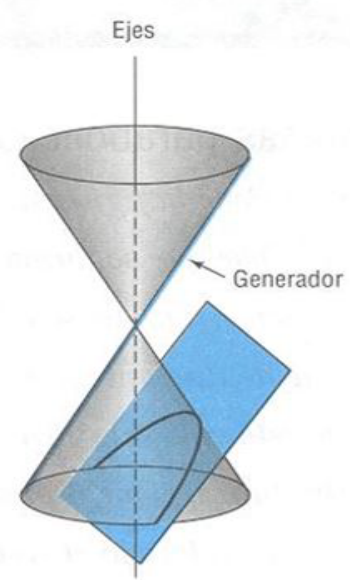

(c) Parábola

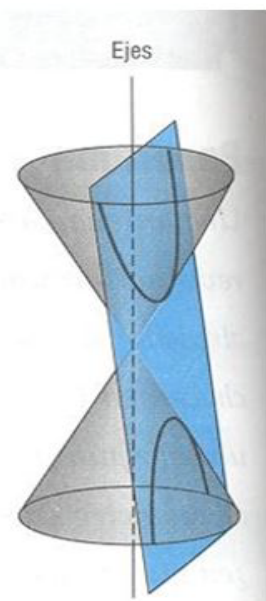

(d) Hipérbola

Imagen 4. Tipos de curva de origen cónico: circulo, elipse, parábola e hipérbola.

Fuente: Ramírez (2011)

De acuerdo a estas características geométricas, es importante resaltar que existe otro tipo de figura, la curva catenaria. En matemáticas y arquitectura se recurre a la palabra catenaria para distinguir la curva que

sigue la forma que adquiere una cadena o cuerda de densidad uniforme y perfectamente flexible sujeta por sus dos extremos y que se encuentra sometida únicamente a las fuerzas de la gravedad de Zárraga (2003). Esta forma es usada en la arquitectura e ingeniería muy resistente a la tracción y compresión en respuesta a las fuerzas generadas por la gravedad.

En la imagen 4 se puede observar como el trazado de la parábola se asemeja mucho al trazado de la catenaria, no obstante, estas curvas son diferentes, pues mientras la parábola está definida por una ecuación cuadrática, en la expresión de la catenaria se incluyen funciones hiperbólicas según lo comentado por de Zárraga (2003). Esta autora también menciona el matemático alemán Joachin Jungius en 1669, que fue capaz de demostrar que una cadena colgante no adoptaba una forma de parábola.
Para comprender las diferencias entre la catenaria y una parábola, se puede decir por un lado que, en el caso de la catenaria, el valor de la tangente tiende a la verticalidad, mientras que en la parábola este valor tiene a una constante (Imagen 5). Esto condiciona que, en la catenaria, para valores infinitos de la y, la x tiende a valores limitados, mientras que en la parábola para los valores infinitos de la y se obtienen valores infinitos de la x. En consecuencia, la fórmula matemática de estas dos curvas tienen similitudes, pero los últimos términos de esta expresión se fórmula $\mathrm{x}$ elevadas a potencias mayores, en el caso de la catenaria. De esta manera se pueden distinguir sus fórmulas:

Ecuación de la catenaria: $y=\alpha \operatorname{Cosh}((x-x 0) / \alpha)$ $+\mathrm{y} 0$.

Ecuación de la parábola: $y=a \cdot x 2+b \cdot x+c$ 

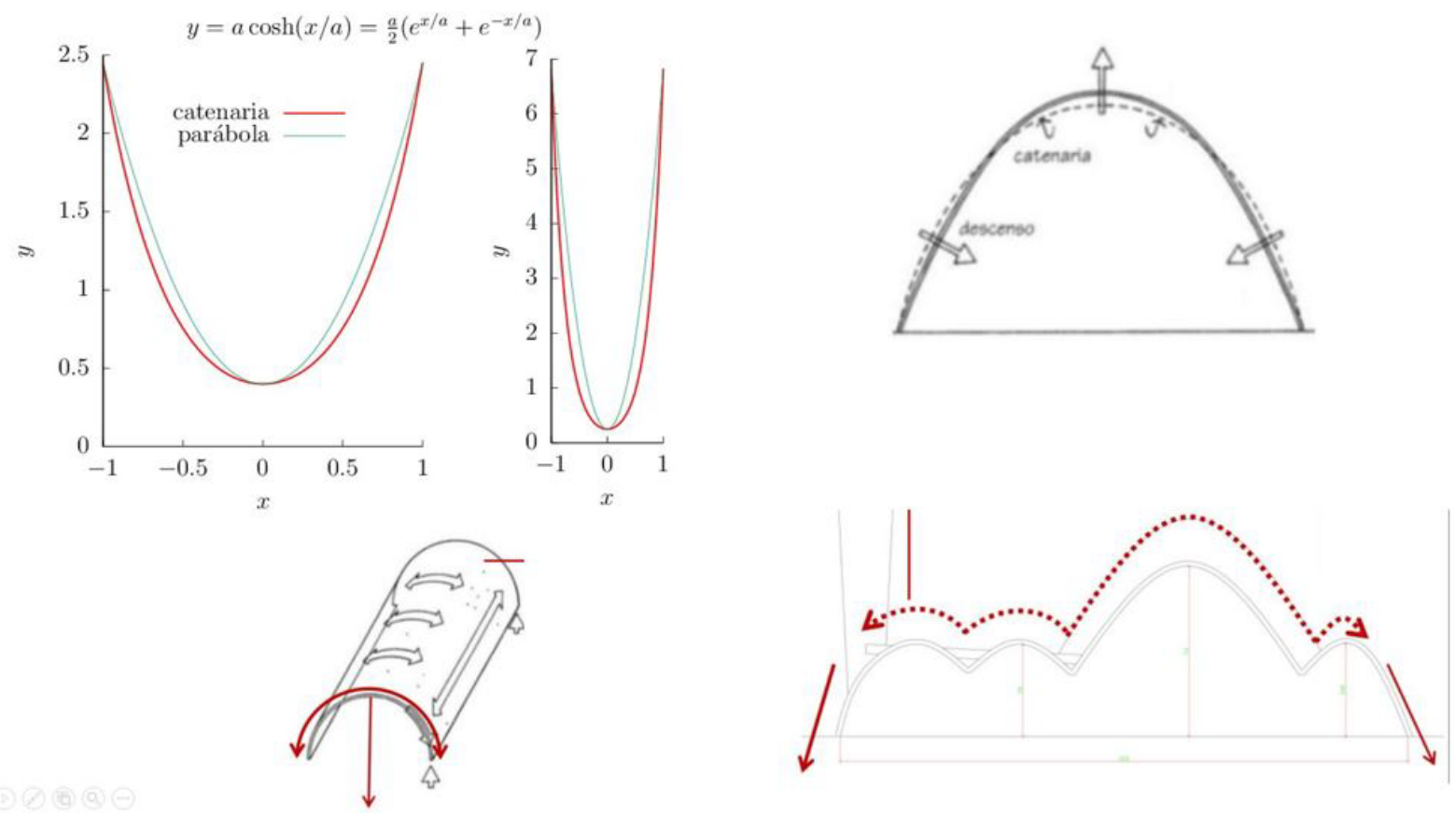

Imagen 5. Comparación de las curvas catenaria y parábola, aplicación grafica a la iglesia de San Francisco de Asís, Brasil

Fuente: Elaboración propia con grafico de Ramírez (2011)

Aplicando este principio en la estructura del caso de estudio, el arco que reproduce exactamente la morfología de la Iglesia de San Francisco de Asís es una curva catenaria invertida, denominada arco catenario. Es decir, matemáticamente las características de la catenaria se conservan al invertir su gráfica. De modo que el arco catenario es la forma ideal para sobrellevar su propio peso, como se presenta en este caso. El trabajo estructural, no presenta fuerzas de cizalla significativas en las uniones y la fuerza al apoyo se transmite a lo largo de la línea del arco. Posee la ventaja que cuando mayor es la altura, más pequeño es el empuje horizontal en los puntos de arranque, con lo que se pueden obtener grandes alturas con mínimos empujes laterales, de ahí la estructura más alta posee mayor resistencia en sus laterales. La transmisión de esfuerzos y presiones hacia los cimientos es la propiedad que hace que los arcos catenarios de esta iglesia no necesiten apoyos laterales para sustentarse.

\section{Conclusión}

El Movimiento Litúrgico incorpora los avances de la modernidad, reafirmado la fe en afinidad con el desarrollo científico y tecnológico. Por otra parte, el movimiento arquitectónico moderno, también formula el propósito de renovación, ya que, se encamino a la austeridad y la simplificación constructiva haciendo uso de la geometría. Con este contexto, se presenta la iglesia de San Francisco de Asís, como un ejemplo tipológico en Latinoamérica. No solo por su innovación tecnología al aplicar el arco catenario en el perfil de hormigón, sino por la conceptualización de las iniciativas del Movimiento Litúrgico en la espacialidad del recinto sagrado, teniendo en cuenta su fecha de inauguración, como sello de su sentido vanguardista. De donde resulta, una planta absolutamente libre de apoyos para facilitar la integración de la zona presbiteral con la audiencia, por tanto, extender el poder del altar a cada uno de los fieles. Luego, facilita la experiencia mística, despreciando la concepción de mero objeto espacial, para vincularse como mediador del hábito 
contemplativo incluso también con el uso de formas geométricas relacionadas con el origen cónico.

De este modo, la geometría estructural se consigue con una técnica constructiva propia de la modernidad, al invertir la curva catenaria, conservando las características matemáticas. El denominado arco catenario, como se le conoce, es la forma ideal para un cuerpo que se soporta a sí mismo. Cuando está construido de elementos individuales cuyas superficies son perpendiculares a la curva del arco, cuyos esfuerzos y cargas son directamente llevados a los cimientos. Su estabilidad no disminuye al crecer en altura, por el contrario, es una forma geométrica muy estable sin que se requieran apoyos complementarios.

\section{Referencias}

Benévolo, Leonardo. (1980) Historia de la Arquitectura moderna. Barcelona. Gustavo Gili, S.A

Díaz, Bárbara y Podetti, Ramiro. «Protestantismo y Concilio Vaticano II: una original tesis de Alberto Methol Ferré». Franciscanum173, Vol. 62 (2020): 1-18

Delgado, A., Díaz, Y. \& Vergel, M. (2018). El paisaje Arquitectónico y sonoro del campanario de la Catedral de San José de Cúcuta. Revista Logos Ciencia \& Tecnología.vol.11 N 1, enero marzo 2.019 P52-60v

Díaz Umaña, Y.., Delgado Rojas, J. A.., \& Vergel Ortega, M. (2021). Representaciones socioespaciales, en la memoria histórica de la catedral y su entorno urbano en San José de Cúcuta. Revista Boletín Redipe, 10(4), 376-387. https://doi.org/10.36260/rbr.v10i4.1277

Díaz, Y., Vergel, M. y Delgado, R. (2020). Modelo geométrico y arquitectónico de la Cúpula Mayor En San José De Cúcuta». Revista Boletín Redipe 9, 3 (2020): 160-166. DOI. https://revista.redipe. org/index.php/1/article/view/941

Duque, Karina. (2012). Clásicos de Arquitectura: Iglesia de Pampulha / Oscar Niemeyer. ArchDaily. Visto en : https:/www.archdaily.co/ co/02-182625/clasicos-de-arquitectura-iglesiade-pampulha-oscar-niemeyer

Flores, Nicolas y Oliva, Gerardo (2016). Arquitectura sustentable con definición de patrones de comportamiento estructurales en materiales y sistemas de materiales actuales. Tlamati Sabiduria, Volumen 7 Número Especial 2 visto en: http://tlamati.uagro.mx/t7e2/60.pdf

Heidegger Martin. (1993). Construir, habitar y pensar. Santiago de chile: editorial Universitaria

Renato De Fusco. (1992). Historia de la Arquitectura contemporánea. Madrid: Celeste Ediciones

León, Leticia (2019). Espacios Sagrados después del Concilio Vaticano II: Templos católicos en Morelia. Actas del congreso Internacional de arquitectura Religiosa contemporánea 4. 2015, 102-107. Consulta en noviembre 102019: https:// dialnet.unirioja.es/descarga/articulo/5849038. pdf

Loos Adolf. "Ornamento y delito". (2019). paperback $n^{\circ} 7$. ISSN 1885-8007. 1908. Consulta en diciembre 3 2019: http://www. paperback.es/ articulos/loos/ornamento.pdf

Navarro Sánchez, Rosana; Castañeda, Fredy Humberto; Peña Esquivel, William Augusto y Acero Montañez, José Ricardo. (2018). «El acontecer del misterio en el silencio, la contemplación y representación». Franciscanum170, Vol. 1x (2018): 183-214

Philippou, Styliane (2013). El modernismo radical de Oscar Niemeyer. Arquitectura y Urbanismo, vol. XXXIV, no 2, mayo-agosto, 2013, p. 5-26, Visto en: ttp://scielo.sld.cu/pdf/au/v34n2/ au020213.pdf

Ramirez, Jaime (2011). HISTORIA DE LAS CONICAS Y LA GEOMETRIA ANALITICA. Gramir 33. visto en: https://gramir33.webnode. es/a $10 \% \mathrm{C} 2 \% \mathrm{~B} 0 /$ geometria-analitica/conicas/

Sabino, Carlos (2008). El proceso de Investigación. Caracas: 17 Editorial Panapo

Téllez, German (1977). Crítica \& Imagen. Bogotá: ESCALA Fondo Editorial

Vallespín, Aurelio; Hernández, Luis y Cervero Noelia (2019). El espacio Gótico según Hans 
Jantzen y la evolución de las vidrieras. Expresión gráfica arquitectónica Universidad Politécnica de Valencia. (2015):238- 247. Consulta en noviembre 26 2019: https://doi.org/10.4995/ ega.2015.3671

de Zárraga Mata, Susana (2003). LA CATENARIA EN. ARQUITECTURA.El cálculo de estructuras en la obra de Gaudí. Ingenieria civil 2003; 129: 121-33 